\title{
Hyperfine Interactions in Titanates: Study of Orbital Ordering and Local Magnetic Properties ${ }^{1}$
}

\author{
P. A. Agzamova, Yu. V. Leskova, and A. E. Nikiforov \\ Ural Federal University, Yekaterinburg, 620002 Russia \\ e-mail: polina_agzamova@usu.ru
}

Received July 2, 2012

\begin{abstract}
Hyperfine magnetic fields induced on the nuclei of nonmagnetic ions ${ }^{139} \mathrm{La}^{\text {and }}{ }^{89} \mathrm{Y}_{\text {in }} \mathrm{LaTiO}_{3}$ and $\mathrm{YTiO}_{3}$, respectively, have been microscopically calculated. The dependence of the hyperfine fields on the orbital and magnetic structures of the compounds under study has been analyzed. The comparative analysis of the calculated and known experimental data confirms the existence of the static orbital structure in lanthanum and yttrium titanates.
\end{abstract}

DOI: $10.1134 / \mathrm{S} 1063776113050166$

\section{INTRODUCTION}

Oxides of transition metals with orbital degeneracy are on the focus of attention of researchers. "Orbital physics" [1] appeared as a new field in the solid state physics that describes the unusual properties of these materials taking into account features caused by orbital degeneracy (orbital structure, Jahn-Teller effect, orbitally dependent exchange interaction). Perovskite oxides $\mathrm{RMO}_{3}$ (where $\mathrm{R}=\mathrm{Y}, \mathrm{La}$ and other trivalent rare-earth ions and $\mathrm{M}$ is a $3 d$ ion) are typical objects for this field. Titanates $\mathrm{RTiO}_{3}$ belonging to this class of compounds seem to be key materials for the study of the strong coupling of the lattice, spin, and orbital degrees of freedom.

Titanates with one $d$ electron in the $t_{2 g}$ shell are convenient objects for answer to the questions: whether a static orbital structure [2, 3] exists in the titanium sublattice or the orbital-liquid state is implemented [4, 5], what is the role of Jahn-Teller distortions, why $\mathrm{LaTiO}_{3}$ is a G-type antiferromagnet with $T_{\mathrm{N}}=140 \mathrm{~K}$ [6], whereas isostructural $\mathrm{YTiO}_{3}$ [7] is a ferromagnet with $T_{\mathrm{C}}=25 \mathrm{~K}$.

The first attempt to clarify the microscopic nature of the unusual properties of titanates was made by Khalliullin and Maekawa [4]. They proposed the orbital liquid model that explains the reduction of the magnetic moment of titanium of about $0.5 \mu_{\mathrm{B}}$ in $\mathrm{LaTiO}_{3}[5]$ and an isotropic spectrum of spin waves [8]. However, the model contradicts the NMR experimental results for ${ }^{47,49} \mathrm{Ti}$ [9], some calculations in the crystal-field model [3, 10], and band calculations [11]. The results of calculations show that the ground level of the $\mathrm{Ti}^{3+}$ ion is an orbital singlet separated from the

\footnotetext{
${ }^{1}$ The article is based on a preliminary report delivered at the 36 th Conference on Low-Temperature Physics (St. Petersburg, July 2-6, 2012).
}

exciting level by a gap of about $0.12-0.25 \mathrm{eV}$, which is too wide for the appearance of orbital fluctuations. Nevertheless, Raman experiments [12] indicate the presence of orbital fluctuations in the ground state. Thus, the role of orbital fluctuations in titanates is incompletely clear.

The orbital structure determines the type and code of the magnetic order in titanate crystals [3, 10]. Orbital fluctuations can change the code of the magnetic structure [10]. The type and code of the magnetic structure are responsible for the magnitude and direction of the induced magnetic field on the nuclei of $\mathrm{La}$ and $\mathrm{Y}$ diamagnetic cations detected in the NMR experiments [13].

The NMR experiments on the ${ }^{47,49} \mathrm{Ti}$ nuclei allow the detection of the local orbital order, whereas NMR on ${ }^{139} \mathrm{La}\left({ }^{89} \mathrm{Y}\right)$ depends on the entire picture of the orbital and magnetic orders. For this reason, investigation of hyperfine fields on La and Y nuclei can provide a more complete picture of the orbital and magnetic orders in titanates.

In this work, the magnetic fields induced on the La and $\mathrm{Y}$ nuclei in $\mathrm{LaTiO}_{3}$ and $\mathrm{YTiO}_{3}$, respectively, are calculated. The dependence of these fields on the crystal, orbital, and magnetic structures of the compounds under study is analyzed. Comparison with the known experimental data is performed.

\section{HYPERFINE FIELDS IN TITANATES AND THEIR RELATION TO THE CRYSTAL, ORBITAL, AND MAGNETIC STRUCTURES}

The problem of the calculation of hyperfine fields induced on the nuclei of nonmagnetic ions in magnetic materials is solved for a long time using various methods [14, 15]. This problem in application to titanates is still topical because there are no theoretical 
works concerning the calculation of induced hyperfine fields in these compounds.

The interatomic hybridization of $d$ electrons in titanium and $s$ and $p$ electrons in lanthanum and yttrium determines the dependence of the hyperfine field on the nucleus of a chosen ion on the distribution of spins and the orientations of orbitals on ions at the neighboring sites of the crystal lattices, i.e., on the crystal, spin, and orbital structures of the material under study. As a result, the form of hyperfine fields depends strongly on a particular type of these structures. According to the crystal structure, titanates belong to the so-called quasi-cubic perovskites. Figure 1 shows the crystal unit cell of titanates, which is assumed cubic in the first approximation. In this approximation, $\mathrm{Ti}^{3+} \mathrm{Jahn}-$ Teller ions ( $3 d^{1}$ configuration) located at the vertices of the cube are in equivalent positions. They correspond to a triply degenerate orbital state with the wavefunctions $\xi, \eta$, and $\zeta$ with the $y z, x z$, and $x y$ symmetries, respectively.

Titanates at low temperatures have a distorted orthorhombic structure [16, 17] because of distortions of oxygen octahedra (displacements of oxygen ions), as well as the displacements of $\mathrm{La}$ or $\mathrm{Y}$ ions (Pnma space group). Owing to the distortion of the crystal structure of titanates, the states of $\mathrm{Ti}$ ions at sites $1,2,3$, and 4 are nonequivalent and are transferred to each other under the action of Pnma symmetry operation (Fig. 1). The wavefunctions of the ground states of $\mathrm{Ti}^{3+}$ ions in different positions (Pnma) calculated in $[10,18]$ are given by the expressions

$$
\begin{gathered}
\psi_{1}\left(\mathrm{LaTiO}_{3}\right)=m \xi+n \eta+p \zeta, \\
\psi_{2}\left(\mathrm{LaTiO}_{3}\right)=-m \xi-n \eta+p \zeta, \\
\psi_{3}\left(\mathrm{LaTiO}_{3}\right)=n \xi+m \eta+p \zeta, \\
\psi_{4}\left(\mathrm{LaTiO}_{3}\right)=-n \xi-m \eta+p \zeta, \\
m=-0.612, \quad n=-0.449, \quad p=0.651 ; \\
\psi_{1}\left(\mathrm{YTiO}_{3}\right)=-m \xi+p \zeta, \\
\psi_{2}\left(\mathrm{YTiO}_{3}\right)=m \xi+p \zeta, \\
\psi_{3}\left(\mathrm{YTiO}_{3}\right)=-m \eta+p \zeta, \\
\psi_{4}\left(\mathrm{YTiO}_{3}\right)=m \eta+p \zeta, \\
m=0.579, \quad p=0.764 .
\end{gathered}
$$

The magnetic structure of titanates is also foursublattice. It appeared that, instead of average spins of sublattices $\mathbf{S}_{1}, \mathbf{S}_{2}, \mathbf{S}_{3}$, and $\mathbf{S}_{4}$, it is convenient to perform particular calculations with their linear combinations expressed in terms of the vectors of magnetic structures:

$$
\begin{array}{ll}
\mathbf{F}=\mathbf{S}_{1}+\mathbf{S}_{2}+\mathbf{S}_{3}+\mathbf{S}_{4}, & \mathbf{A}=\mathbf{S}_{1}-\mathbf{S}_{2}+\mathbf{S}_{3}-\mathbf{S}_{4}, \\
\mathbf{G}=\mathbf{S}_{1}-\mathbf{S}_{2}-\mathbf{S}_{3}+\mathbf{S}_{4}, & \mathbf{C}=\mathbf{S}_{1}+\mathbf{S}_{2}-\mathbf{S}_{3}-\mathbf{S}_{4} .
\end{array}
$$

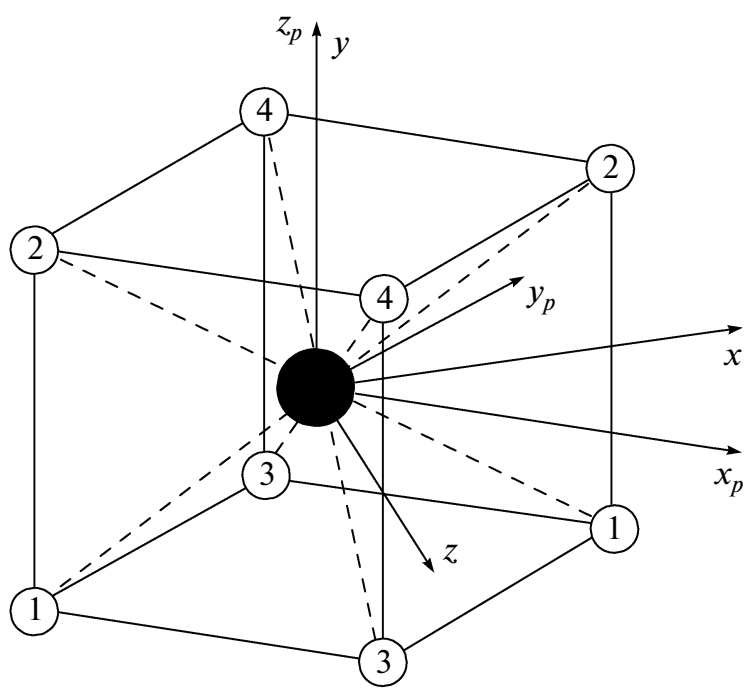

Fig. 1. Crystal structure of titanates: Ti ions at vertices; R ion $(\mathrm{R}=\mathrm{La}, \mathrm{Y})$ at the center; digits mark sublattices; $\left(x_{p}\right.$, $\left.y_{p}, z_{p}\right)$ is the pseudocubic coordinate system; and $(x, y, z)$ is the orthorhombic coordinate system.

In particular, the components $H_{n}^{\alpha}$ of hyperfine fields on ${ }^{139} \mathrm{La}$ and ${ }^{89} \mathrm{Y}$ nuclei are conveniently expressed in terms of the components of the vectors $\mathbf{F}, \mathbf{C}, \mathbf{A}$, and $\mathbf{G}$ as

$$
H_{n}^{\alpha}=a_{\alpha \beta} A_{\beta}+g_{\alpha \beta} G_{\beta}+c_{\alpha \beta} C_{\beta}+f_{\alpha \beta} F_{\beta},
$$

where $a_{\alpha \beta}, g_{\alpha \beta}, c_{\alpha \beta}$, and $f_{\alpha \beta}$ are the hyperfine coupling constants, which are tensors whose symmetry is determined by the symmetry of the environment of the nucleus under consideration. The position of a lanthanum ion is described by the point symmetry group $C_{s}$. For this reason, in the orthorhombic coordinate system, the tensors $a_{\alpha \beta}, g_{\alpha \beta}, c_{\alpha \beta}$, and $f_{\alpha \beta}$ have the form

$$
\begin{gathered}
a_{\alpha \beta}=\left(\begin{array}{ccc}
0 & a_{x y} & 0 \\
a_{y x} & 0 & a_{y z} \\
0 & a_{z y} & 0
\end{array}\right), \\
g_{\alpha \beta}=\left(\begin{array}{ccc}
0 & g_{x y} & 0 \\
g_{y x} & 0 & g_{y z} \\
0 & g_{z y} & 0
\end{array}\right), \\
c_{\alpha \beta}=\left(\begin{array}{ccc}
c_{x x} & 0 & c_{x z} \\
0 & c_{y y} & 0 \\
c_{z x} & 0 & c_{z z}
\end{array}\right), \\
f_{\alpha \beta}=\left(\begin{array}{ccc}
f_{x x} & 0 & f_{x z} \\
0 & f_{y y} & 0 \\
f_{z x} & 0 & f_{z z}
\end{array}\right) .
\end{gathered}
$$




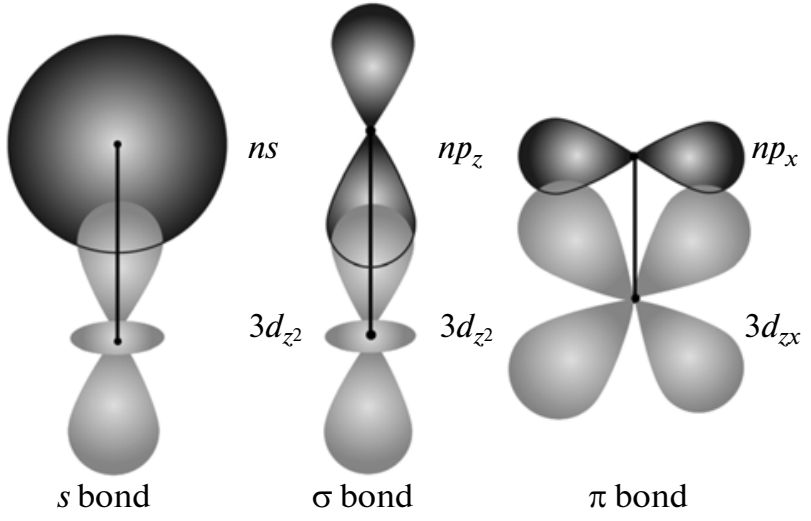

Fig. 2. Scheme of the formation of the $s, \sigma$, and $\pi$ bonds. Light and dark regions indicate Ti orbitals and $\mathrm{R}$ ion orbitals $(\mathrm{R}=\mathrm{La}, \mathrm{Y})$, respectively.

The aim of this work is to calculate the components of the tensors $a_{\alpha \beta}, g_{\alpha \beta}, c_{\alpha \beta}$, and $f_{\alpha \beta}$ for nonmagnetic ions $\mathrm{Y}$ and $\mathrm{La}$ in titanates.

To calculate them, it is necessary to take into account the hybridization of the wavefunctions of filled $s$ and $p$ shells of $Y$ and La ions $\left(\chi_{s}, \chi_{0}, \chi_{ \pm 1}\right)$ with the wavefunctions of the unfilled $d$ shell of Ti ions $\left(d_{0}, d_{ \pm 1}, d_{ \pm 2}\right)$ from the local environment of yttrium and lanthanum.

According to the molecular orbital method, which is usually used to calculate wavefunctions for a pair of ions taking into account interatomic hybridization, the desired functions can be represented in the form

$$
\begin{gathered}
\varphi_{0}=\frac{1}{\sqrt{N_{0}}}\left(d_{0}-\lambda_{S} \chi_{s}-\lambda_{\sigma} \chi_{0}\right), \\
\varphi_{ \pm 1}=\frac{1}{\sqrt{N_{1}}}\left(d_{ \pm 1}-\lambda_{\pi} \chi_{ \pm 1}\right), \\
\varphi_{ \pm 2}=d_{ \pm 2} .
\end{gathered}
$$

The molecular orbitals are constructed taking into account symmetry reasons and the so-called "bonding rules," according to which $s$ and $d_{z^{2}}$ states form an $s$ bond, $p_{z}$ and $d_{z^{2}}$ states form a $\sigma$ bond, and $p_{x}\left(p_{y}\right)$ and $d_{x z}\left(d_{y z}\right)$ states form a $\pi$ bond (Fig. 2). A bond is characterized by the overlapping integrals $\left(\lambda_{S}, \lambda_{\sigma}, \lambda_{\pi}\right)$. The explicit expressions for the overlapping integrals between the radial wavefunctions of $5 s$ and $5 p$ electrons of $\mathrm{La}^{3+}$ [19], $4 s$ and $4 p$ electrons of $\mathrm{Y}^{3+}$, and $3 d$ electrons of $\mathrm{Ti}^{3+}[20]$ are obtained by the method proposed in [21].

\section{SCHEME OF THE CALCULATION \\ OF HYPERFINE FIELDS INDUCED \\ ON THE NUCLEI OF NONMAGNETIC ${ }^{139} \mathrm{La}$ AND ${ }^{89} \mathrm{Y}$ IONS}

The hyperfine interaction of a magnetic ionligand pair in the crystal field can be represented in the form of the effective spin Hamiltonian

$$
H_{\mathrm{HFI}}^{\mathrm{eff}}(\mathbf{r})=\mathbf{I} \cdot A(\mathbf{r}) \cdot \mathbf{s},
$$

where $\mathbf{r}$ is the operator of the coordinates of an electron in the coordinate system with the origin at the point of the nucleus $\mathbf{I}, \mathbf{s}$ is the electron spin operator, and $A$ is the hyperfine interaction operator given by the expression

$$
A=\sum B_{q}^{k} C_{q}^{k}
$$

Here,

$$
C_{k q}(\vartheta, \phi)=\sqrt{\frac{4 \pi}{2 k+1}} Y_{k q}(\vartheta, \phi),
$$

are the weighted spherical harmonics, where $\vartheta$ and $\phi$ are the angular coordinates, $Y_{k q}(\vartheta, \phi)$ are the spherical harmonics, and the parameters $B_{q}^{k}$ are closely related to the crystal and orbital structures of titanates and are determined from the condition

$$
\left\langle\varphi_{m}\left|\hat{H}_{\mathrm{HFI}}\right| \varphi_{m}\right\rangle=\left\langle d_{m}\left|\hat{H}_{\mathrm{HFI}}^{\text {eff }}\right| d_{m}\right\rangle,
$$

where $\hat{H}_{\mathrm{HFI}}$ is the Hamiltonian of the magnetic hyperfine interaction [13] given by the expression

$$
\begin{gathered}
\hat{H}_{\mathrm{HFI}}(\mathbf{r})=2 \mu_{\mathrm{B}} \gamma_{n} \hbar \mathbf{I} \cdot\left\{\frac{\mathbf{l}}{r^{3}}-\frac{\mathbf{s}}{r^{3}}\right. \\
\left.+\frac{3 \mathbf{r}(\mathbf{s} \cdot \mathbf{r})}{r^{5}}+\frac{8}{3} \pi \mathbf{s} \delta(\mathbf{r})\right\} .
\end{gathered}
$$

Hamiltonian (6) is represented in the local coordinate system with the $z$ axis oriented along the $\mathrm{La}(\mathrm{Y})-\mathrm{Ti}$ bond. The analysis of the crystal structure shows that the angles between this bond and the $z$ and $x$ axes are $\theta$ and $\varphi$, respectively. Magnetic titanium ions that contribute to the hyperfine field on the nucleus of a lanthanum or yttrium nonmagnetic ion are located at the sites of four sublattices (Fig. 1). The orbitals of each of four sublattices are specifically ordered, which affects the hyperfine fields of nonmagnetic ions.

Formulas (6)-(10) provide the following expressions for the isotropic and anisotropic magnetic hyperfine coupling constants:

$$
\begin{aligned}
A^{\mathrm{is}} & =\left\langle\psi _ { \mathrm { Ti } } \left(\frac{1}{5}+\sum_{q} C_{2 q}^{*}(\theta, \phi) C_{2 q}(\vartheta, \varphi)\right.\right. \\
& \left.+\frac{9}{5} \sum_{q} C_{4 q}^{*}(\theta, \phi) C_{4 q}(\vartheta, \varphi)\right) a_{S}\left|\psi_{\mathrm{Ti}}\right\rangle,
\end{aligned}
$$




$$
\begin{gathered}
A^{\text {an }}=\left\langle\psi_{\mathrm{Ti}}\right| \frac{1}{5}\left(a_{\sigma}-a_{\pi}\right)+\left(a_{\sigma}-\frac{a_{\pi}}{2}\right) \\
\times \sum_{q} C_{2 q}^{*}(\theta, \phi) C_{2 q}(\vartheta, \varphi) \\
+\frac{3}{5}\left(3 a_{\sigma}+2 a_{\pi}\right) \sum_{q} C_{4 q}^{*}(\theta, \phi) C_{4 q}(\vartheta, \varphi)\left|\psi_{\mathrm{Ti}}\right\rangle .
\end{gathered}
$$

The ranks of the spherical harmonics are limited because we consider a $d$ electron. In Eqs. (11) and (12), $\psi_{\mathrm{Ti}}$ are the wavefunctions of the orbital states of titanium ions in $\mathrm{LaTiO}_{3}$ and $\mathrm{YTiO}_{3}$ given by Eqs. (1), and $a_{S}, a_{\sigma}$, and $a_{\pi}$ are the isotropic and anisotropic hyperfine coupling constants associated with the hybridization of the $s, p_{\sigma}$, and $p_{\pi}$ shells, respectively, of La or Y ions with the $d$ shell of the Ti ion (Fig. 2).

The isotropic hyperfine coupling constant is

$$
a_{S}=\left(N_{0}^{-1}\right)^{2} \lambda_{S}^{2} a_{S}^{(0)}
$$

where

$$
a_{S}^{(0)}=\frac{16}{3} \pi \mu_{\mathrm{B}} \gamma_{n} \hbar\left|\varphi_{n s}(0)\right|^{2}
$$

is the contribution to $a_{S}$ from a single $4 s$ electron of the $\mathrm{Y}$ ion and a $5 s$ electron of La.

The anisotropic hyperfine coupling constants are

$$
a_{\sigma}=\left(N_{1}^{-1}\right)^{2} \lambda_{\sigma}^{2} a_{p}^{(0)}, \quad a_{\pi}=\left(N_{1}^{-1}\right)^{2} \lambda_{\pi}^{2} a_{p}^{(0)},
$$

where

$$
a_{p}^{(0)}=\frac{4}{5} \pi \mu_{\mathrm{B}} \gamma_{n} \hbar\langle r\rangle_{n p}^{-3}
$$

is the contribution to $a_{\sigma}$ and $a_{\pi}$ from a single $4 p$ electron of $\mathrm{Y}$ and a $5 p$ electron of La.

Formulas (11) and (12), inclusion of the rotation of the $\mathrm{La}(\mathrm{Y})-\mathrm{Ti}$ bond by angles $(\theta, \varphi)$, and inclusion of contributions to the hyperfine interaction on the nuclei of La and Y nonmagnetic ions from eight nearest magnetic Ti ions make it possible to represent the spin Hamiltonian of the local magnetic hyperfine fields induced on ${ }^{139} \mathrm{La}$ and ${ }^{89} \mathrm{Y}$ given by Eq. (6) in the form

$$
\begin{gathered}
\hat{H}_{\mathrm{HFI}}^{\mathrm{eff}}=\sum_{n=1}^{8}\left\{A^{\mathrm{is}}\left(\mathbf{I} \cdot \mathbf{S}^{n}\right)+A^{\mathrm{an}} \frac{3}{2}\right. \\
\times\left\{\left(\cos ^{2} \theta_{n}-\frac{1}{3}\right)\left(3 I_{z} S_{z}^{n}-\left(\mathbf{I} \cdot \mathbf{S}^{n}\right)\right)+\sin \left(2 \theta_{n}\right)\right. \\
\times\left[\cos \varphi_{n}\left(I_{x} S_{z}^{n}+I_{z} S_{x}^{n}\right)+\sin \varphi_{n}\left(I_{y} S_{z}^{n}+I_{z} S_{y}^{n}\right)\right] \\
+\sin ^{2} \theta_{n}\left[\cos \left(2 \varphi_{n}\right)\left(I_{x} S_{x}^{n}-I_{y} S_{y}^{n}\right)\right. \\
\left.\left.+\sin \left(2 \varphi_{n}\right)\left(I_{x} S_{y}^{n}+I_{y} S_{x}^{n}\right)\right]\right\}
\end{gathered}
$$

Here, $A^{\text {is }}$ and $A^{\text {an }}$ are given by Eqs. (11) and (12), respectively, the index $n$ means that summation is performed over the nearest magnetic neighbors surrounding the nonmagnetic ion, and $\mathbf{S}^{n}$ is the average value of the spins of sublattices. The expression for the induced magnetic hyperfine field given by Eq. (3) is obtained after the transition from $\mathbf{S}^{n}$ to the magnetic-structure vectors $\mathbf{F}, \mathbf{C}, \mathbf{A}$, and $\mathbf{G}$.

\section{RESULTS AND DISCUSSION}

Using the scheme for the calculation of hyperfine fields induced on the nuclei of nonmagnetic lanthanum and yttrium ions in $\mathrm{LaTiO}_{3}$ and $\mathrm{YTiO}_{3}$, respectively, we calculate the components of magnetic hyperfine interaction tensors $a_{\alpha \beta}, g_{\alpha \beta}, c_{\alpha \beta}$, and $f_{\alpha \beta}$ given by Eqs. (4) taking into account the effect of the crystal, orbital, and magnetic structures of titanates.

The spin Hamiltonian of induced magnetic hyperfine fields calculated in the work depends only on three parameters $a_{S}, a_{\sigma}$, and $a_{\pi}$. The set of the parameters $a_{S}, a_{\sigma}$, and $a_{\pi}$ were estimated by comparing the experimental spectrum [13], as well as using Eqs. (14) and (15) and atomic wavefunctions of the ions [19, 20] involved in the hyperfine interaction. The comparative analysis of the results indicates that the microscopic calculations with the use of atomic wavefunctions underestimate the parameters $a_{S}, a_{\sigma}$, and $a_{\pi}$ as compared to the estimate from the comparison with the experimental spectrum. However, $a_{S} / a_{\sigma}=a_{S} / a_{\pi}=0.1$.

The group-theory analysis of the magnetic structure for crystals with space group Pnma gives the following possible types of magnetic structures: $\left(A_{x}, F_{y}\right.$, $\left.G_{z}\right),\left(G_{x}, C_{y}, A_{z}\right),\left(F_{x}, A_{y}, C_{z}\right)$, and $\left(C_{x}, G_{y}, F_{z}\right)$ [22]. The magnetic structures for lanthanum and yttrium titanates for the static orbital state and state with strong orbital fluctuations were determined in [10]. The calculations show that the magnetic structure of $\mathrm{LaTiO}_{3}$ and $\mathrm{YTiO}_{3}$ in the orbital order state has the code $\left(A_{x}\right.$, $\left.F_{y}, G_{z}\right)$. The magnetic structure of $\mathrm{LaTiO}_{3}$ and $\mathrm{YTiO}_{3}$ in the state with strong orbital fluctuations has the codes $\left(G_{x}, C_{y}, A_{z}\right)$ and $\left(F_{x}, A_{y}, C_{z}\right)$, respectively. The largest contribution in both states of lanthanum and yttrium titanates comes from the $G$ and $F$ components, respectively. Thus, the analysis of the code of the magnetic structure of magnets can distinguish the static orbital state and the state with strong orbital fluctuations, which is manifested in nuclear magnetic resonance.

In the orbital order state, according to the magnetic structure $\left(A_{x}, F_{y}, G_{z}\right)$, only the component of the induced hyperfine field given by Eq. (3) that is directed along the orthorhombic $b$ axis ( $y$ axis in Fig. 1) is nonzero:

$$
\begin{gathered}
{ }^{89} H_{y}^{\text {is }}=-\frac{1}{{ }^{89} \gamma_{n} \hbar} \times 0.582 a_{S} F_{y}, \\
{ }^{89} H_{y}^{\mathrm{an}}=-\frac{1}{{ }^{89} \gamma_{n} \hbar}\left[\left(-0.087 a_{\pi}-0.432 a_{\sigma}\right) A_{x}\right.
\end{gathered}
$$




$$
\begin{gathered}
\left.+\left(0.008 a_{\pi}+0.040 a_{\sigma}\right) F_{y}+\left(0.015 a_{\pi}+0.067 a_{\sigma}\right) G_{z}\right], \\
{ }^{139} H_{y}^{\text {is }}=-\frac{1}{{ }^{139} \gamma_{n} \hbar} \times 0.693 a_{S} F_{y}, \\
{ }^{139} H_{y}^{\text {an }}=-\frac{1}{{ }^{139} \gamma_{n} \hbar}\left[\left(0.030 a_{\pi}+0.317 a_{\sigma}\right) A_{x}\right. \\
\left.+\left(-0.006 a_{\pi}-0.063 a_{\sigma}\right) F_{y}+\left(-0.040 a_{\pi}-0.219 a_{\sigma}\right) G_{z}\right] .
\end{gathered}
$$

The analysis of the calculations of induced hyperfine fields shows that the isotropic hyperfine field for both $\mathrm{LaTiO}_{3}$ and $\mathrm{YTiO}_{3}$ is determined only by the $F$ component. The other components do not contribute to the isotropic hyperfine field owing to the symmetry of nonmagnetic ions ( $\mathrm{La}$ and $\mathrm{Y}$ ). The contribution of the $F_{y}$ component for $\mathrm{LaTiO}_{3}$ is small $(\approx 0.006)$. For this reason, the contribution from the isotropic hyperfine field is almost zero in agreement with the experiment [13]. However, there is a contribution from the anisotropic hyperfine field, where the contribution of the $G_{z}$ component, which is the main contribution for antiferromagnetic $\mathrm{LaTiO}_{3}$, is approximately 0.999. Consequently, a nonzero local hyperfine magnetic field having anisotropic nature appears on the nucleus of the $\mathrm{La}$ ion. The hyperfine field induced on the nucleus of the nonmagnetic $\mathrm{Y}$ ion in $\mathrm{YTiO}_{3}$ has isotropic nature because the contribution from the $F_{y}$ component determining the isotropic hyperfine field dominates in the formation of the static magnetic structure $(\approx 0.993)$. The anisotropic hyperfine interaction makes an additional contribution to the hyperfine field on the ${ }^{89} \mathrm{Y}$ nucleus because of the mixing of the contributions from the $A_{x}$ component $(\approx 0.115)$ and $G_{z}$ component $(\approx 0.008)$ of the magnetic field.

In the state with strong orbital fluctuations, according to the type of the magnetic structure in $\mathrm{LaTiO}_{3}$, only the $H_{y}$ component of the induced hyperfine magnetic structure is nonzero:

$$
\begin{gathered}
{ }^{139} H_{y}^{\mathrm{is}}=-\frac{1}{{ }^{139} \gamma_{n} \hbar}\left(-0.305 a_{S} C_{y}\right), \\
{ }^{139} H_{y}^{\mathrm{an}}=-\frac{1}{{ }^{139} \gamma_{n} \hbar}\left[\left(0.071 a_{\pi}+0.467 a_{\sigma}\right) G_{x}\right. \\
\left.+\left(-0.006 a_{\pi}-0.047 a_{\sigma}\right) C_{y}+\left(0.012 a_{\pi}+0.082 a_{\sigma}\right) A_{z}\right] .
\end{gathered}
$$

According to the code of the magnetic structure in $\mathrm{YTiO}_{3}$, only the $H_{x}$ and $H_{z}$ components of the induced hyperfine magnetic field are nonzero

$$
\begin{gathered}
{ }^{89} H_{x}^{\text {is }}=-\frac{1}{{ }^{89} \gamma_{n} \hbar} \times 0.582 a_{S} F_{x}, \\
{ }^{89} H_{z}^{\text {is }}=-\frac{1}{{ }^{89} \gamma_{n} \hbar}\left(-0.080 a_{S} C_{z}\right), \\
{ }^{89} H_{x}^{\text {an }}=-\frac{1}{{ }^{89} \gamma_{n} \hbar}\left[\left(0.056 a_{\pi}+0.114 a_{\sigma}\right) F_{x}\right.
\end{gathered}
$$$$
\left.+\left(0.030 a_{\pi}+0.316 a_{\sigma}\right) A_{y}+\left(-0.005 a_{\pi}-0.033 a_{\sigma}\right) G_{z}\right],
$$$$
{ }^{89} H_{z}^{\mathrm{an}}=-\frac{1}{{ }^{89} \gamma_{n} \hbar}\left[\left(-0.011 a_{\pi}-0.069 a_{\sigma}\right) F_{x}\right.
$$$$
\left.+\left(-0.033 a_{\pi}-0.240 a_{\sigma}\right) A_{y}+\left(-0.148 a_{\pi}+0.390 a_{\sigma}\right) G_{z}\right] \text {. }
$$

The analysis of the calculations shows that the isotropic hyperfine field for $\mathrm{LaTiO}_{3}$ determined only by the $C_{y}$ component $(\approx 0.088)$ is almost zero and the hyperfine magnetic field induced on the ${ }^{139} \mathrm{La}$ nucleus has anisotropic nature, where the main contribution comes from the $G_{x}$ component ( $\left.\approx 0.996\right)$. The hyperfine magnetic field induced on the ${ }^{89} \mathrm{Y}$ nucleus in $\mathrm{YTiO}_{3}$ is determined by the $H_{x}$ and $H_{z}$ components. Nevertheless, the calculations show that this field has isotropic nature determined by the contribution from the $F_{x}$ component of the magnetic field $(\approx 0.999)$ with an additional contribution associated with the anisotropic hyperfine field.

The discussed features of the hyperfine magnetic fields induced on the nuclei of nonmagnetic La and $\mathrm{Y}$ ions in the $\mathrm{LaTiO}_{3}$ and $\mathrm{YTiO}_{3}$ compounds in the orbital order state and the state with strong orbital fluctuations affect the behavior of NMR frequencies, which is qualitatively different in these orbital states, which can be observed in NMR on single crystals. Experimental works on NMR in titanates on the nuclei of nonmagnetic ions in single crystals are absent. However, NMR experiments were performed on polycrystalline samples [13, 23].

Case of $\mathrm{LaTiO}_{3}$. The calculation of the parameters of nuclear quadrupole interaction and magnetic fields induced on the lanthanum ion makes it possible to plot the NMR spectrum of the lanthanum ion (Fig. 3). In the absence of external magnetic field, the spectrum consists of two lines centered at the frequencies of 7 and $11 \mathrm{MHz}$ in agreement with the experimental spectrum obtained in [13]. The position and shape of the NMR spectral line on the nucleus of the nonmagnetic lanthanum ion, where the nucleus has the spin $I=7 / 2$, are determined by the electric quadrupole and magnetic hyperfine interactions. The nonzero magnetic field induced on lanthanum, which is no more than $1 \mathrm{kOe}$ in the absence of external magnetic field, leads to the broadening of lines and a complex shape of the 7-MHz spectral line, where the peak is split.

Case of $\mathrm{YTiO}_{3}$. The inverse situation is observed in yttrium titanate [13]. The ferromagnetic structure of the compound leads to a strong increase in the component of the induced magnetic field associated with the polarization of the $s$ shell of yttrium. Its magnitude is approximately estimated as $7 \mathrm{kOe}$ (cf. an experimental value of $10.3 \mathrm{kOe}$ ). The component of the magnetic field induced on the yttrium ion associated with the polarization of the $p$ shells has the opposite sign and its magnitude does not exceed $1 \mathrm{kOe}$. A change in the induced magnetic fields leads to a significant shift of 
Amplitude, arb. units

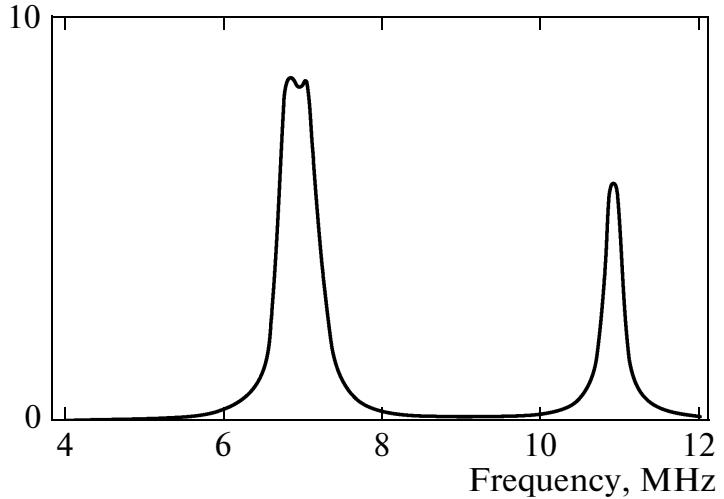

Fig. 3. Spectrum of NMR on ${ }^{139} \mathrm{La}$ in zero magnetic field theoretically calculated with the set of the parameters of the nuclear quadrupole interaction ${ }^{139} v_{Q}=3.70 \mathrm{MHz}$ and $\eta=0.61$ and the parameters of the hyperfine magnetic interaction $a_{S}=0.5 \mathrm{MHz}, a_{\sigma}=5 \mathrm{MHz}$, and $a_{\pi}=$ 4.5 $\mathrm{MHz}$.

the yttrium line in the NMR spectrum observed experimentally [13].

\section{CONCLUSIONS}

The spin Hamiltonian of the magnetic hyperfine interaction depending only on three parameters $a_{S}, a_{\sigma}$, and $a_{\pi}$ has been obtained, the magnetic fields induced on the nuclei of nonmagnetic lanthanum and yttrium ions in the $\mathrm{LaTiO}_{3}$ and $\mathrm{YTiO}_{3}$ compounds have been calculated, and the dependences on the crystal, orbital, and magnetic structures have been analyzed. The analysis of the experimental and calculated data shows that the known NMR experiments on ${ }^{139} \mathrm{La}$ and ${ }^{89} \mathrm{Y}$ nuclei in polycrystals confirm the existence of the static orbital structure in lanthanum and yttrium titanates. Experiments on single crystals can provide information on small magnetization components that are involved in the formation of the magnetic structure of titanates, which is in turn due to the orbital structure.

\section{ACKNOWLEDGMENTS}

This work was supported by the Russian Foundation for Basic Research (project no. 11-02-00093).

\section{REFERENCES}

1. B. Keimer and A. Oles, New J. Phys. 6, E05 (2004).

2. M. Mochizuki and M. Imada, New J. Phys. 6, 154 (2004).

3. R. Schmitz, O. Entin-Wohlman, A. Aharony, A. B. Harris, and E. Müller-Hartmann, Phys. Rev. B: Condens. Matter 71, 144412 (2005).
4. G. Khaliullin and S. Maekawa, Phys. Rev. Lett. 85, 3950 (2000).

5. G. Khaliullin, Prog. Theor. Phys. Suppl. 160, 155 (2008).

6. Y. Okada, T. Arima, Y. Tokura, C. Murayama, and N. Mori, Phys. Rev. B: Condens. Matter 48, 9677 (1993).

7. Y. Taguchi, Y. Tokura, T. Akima, and F. Inaba, Phys. Rev. B: Condens. Matter 48, 511 (1993).

8. B. Keimer, D. Casa, A. Ivanov, J. W. Lynn, M. V. Zimmermann, J. P. Hill, D. Gibbs, Y. Taguchi, and Y. Tokura, Phys. Rev. Lett. 85, 3946 (2000).

9. T. Kiyama and M. Itoh, Phys. Rev. Lett. 91, 167202 (2003).

10. A. A. Mozhegorov, A. V. Larin, A. E. Nikiforov, L. E. Gontchar, and A. V. Efremov, Phys. Rev. B: Condens. Matter 79, 054418 (2009).

11. I. V. Solovyev, Phys. Rev. B: Condens. Matter 74, 054412 (2006).

12. C. Ulrich, A. Gössling, M. Grüninger, M. Guennou, H. Roth, M. Cwik, T. Lorenz, G. Khaliullin, and B. Keimer, Phys. Rev. Lett. 97, 157401 (2006).

13. M. Itoh, M. Tsuchiya, H. Tanaka, and K. Motoya, J. Phys. Soc. Jpn. 68, 2783 (1999).

14. E. A. Turov and M. P. Petrov, Nuclear Magnetic Resonance in Ferro- and Antiferromagnets (Nauka, Moscow, 1969; Israel Program for Scientific Translations, Jerusalem, 1972).

15. A. Abragam and B. Bleaney, Electron Paramagnetic Resonance of Transition Ions (Clarendon, Oxford, 1970; Mir, Moscow, 1972), Vol. 2.

16. A. C. Komarek, H. Roth, M. Cwik, W.-D. Stein, J. Baier, M. Kriener, F. Bourée, T. Lorenz, and M. Braden, Phys. Rev. B: Condens. Matter 75, 224402 (2007).

17. M. Cwik, T. Lorenz, J. Baier, R. Müller, G. André, F. Bourée, F. Lichtenberg, A. Freimuth, R. Schmitz, E. Müller-Hartmann, and M. Braden, Phys. Rev. B: Condens. Matter 68, 060401(R) (2003).

18. A. A. Mozhegorov, A. E. Nikiforov, A. V. Larin, A. V. Efremov, L. E. Gonchar', and P. A. Agzamova, Phys. Solid State 50 (9), 1795 (2008).

19. A. D. McLean and R. S. McLean, At. Data Nucl. Data Tables 26, 197 (1981).

20. E. Clementi and C. Roetti, At. Data Nucl. Data Tables 14, 177 (1974).

21. M. V. Eremin, Spectroscopy of Crystals (Nauka, Leningrad, 1989), p. 30 [in Russian].

22. E. A. Turov, A. V. Kolganov, V. V. Men'shenin, et al., Symmetry and Physical Properties of Antiferromagnets (Fizmatlit, Moscow, 2000) [in Russian].

23. Y. Furukawa, I. Okamura, K. Kumagai, T. Goto, T. Fukase, Y. Taguchi, and Y. Tokura, Phys. Rev. B: Condens. Matter 59, 10550 (1998).

Translated by R. Tyapaev 